\title{
Treatment for Hearing Loss among the Elderly: Auditory Outcomes and Impact on Quality of Life
}

\author{
Raquel Manrique-Huarte Diego Calavia Alicia Huarte Irujo Laura Girón \\ Manuel Manrique-Rodríguez \\ Otorhinolaryngology Department, University of Navarra Clinic, Pamplona, Spain
}

\section{Key Words}

Presbycusis - Age-related hearing loss - Cochlear implant .

Hearing aid · Elderly · Auditory outcomes · Quality of life

\section{Abstract}

The study aim was to determine the benefit of cochlear implantation and hearing aids in older adults diagnosed with hearing loss and to evaluate the index of depression, anxiety and quality of life after such treatments. A retrospective cohort comprised 117 patients older than 65 years and diagnosed with moderate to profound hearing loss who were included and classified into 2 groups (treated vs. non-treated). A battery of tests including auditory (pure-tone average, disyllabic words in quiet at $65 \mathrm{~dB} \mathrm{SPL}$ ) and findings from a series of questions relevant to quality of life were compared between both groups. Auditory outcomes for disyllabic words were $58.21 \%$ for the cochlear implant-treated group and $82.8 \%$ for the hearing aid-treated group. There was a positive effect on anxiety, depression, health status and quality of life in the cochlear implant group versus the profound hearing loss control group. We conclude that older adults with moderate to profound hearing loss gain benefit

\section{KARGER}

E-Mail karger@karger.com www.karger.com/aud
(C) 2016 The Author(s)

Published by S. Karger AG, Basel 1420-3030/16/0217-0029\$39.50/0

This article is licensed under the Creative Commons AttributionNonCommercial-NoDerivatives 4.0 International License (CC BY NC-ND) (http://www.karger.com/Services/OpenAccessLicense). Usage and distribution for commercial purposes as well as any distribution of modified material requires written permission. from hearing aids or cochlear implants not only in terms of improved hearing function, but also in terms of positive effects on anxiety, depression, health status and quality of life.

(c) 2016 The Author(s)

Published by S. Karger AG, Basel

\section{Introduction}

Hearing loss is one of the most common complaints of sensory dysfunction in older adults. It has been estimated that $25 \%$ of the population between 65 and 75 years old have hearing loss, and this percentage increases up to 70$80 \%$ in the age group 75 years of age and older [Sprinzl and Riechelmann, 2010]. Demographic trends, including greater longevity and growing numbers of older adults, point to the relevance of understanding and managing hearing loss. Recent studies suggest that managing hearing loss is paramount, not only to restore hearing ability, but also to improve social skills, cognitive ability and quality of life.

This study aims to analyze auditory outcomes following treatment with a cochlear implant (CI) or hearing aid 
Fig. 1. Study cohort: 4 subgroup classifications, showing summary characteristics for hearing loss, mean pure-tone average (PTA; 0.5-4 kHz), mean age, and gender. Subgroups included: untreated moderatesevere SNHL (MS-SNHL), untreated profound SNHL (P-SNHL), and treated groups, i.e. $\mathrm{CI}$ and HA users. $\mathrm{F}=$ Female; $\mathrm{M}=$ male.

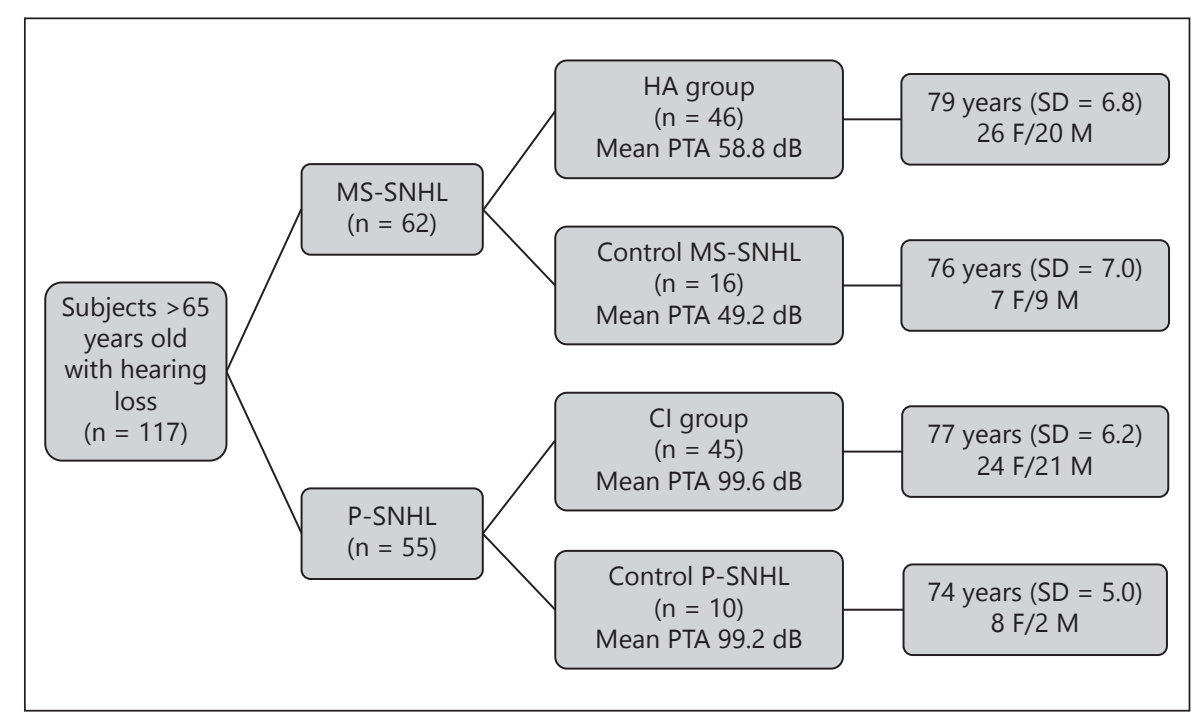

(HA) in patients older than 65 years diagnosed with hearing loss, and to evaluate the index of depression, anxiety and quality of life compared to control groups with similar characteristics who received no treatment for their hearing loss.

\section{Materials and Methods}

A retrospectively examined hearing-impaired cohort of elderly patients routinely seen in our clinic underwent a battery of prospective assessments to measure the impact of hearing loss on their overall well-being. All study subjects signed a written informed consent form to confirm their voluntary participation in the study.

\section{Subjects}

A subgroup of 117 subjects from our routinely treated clinical population of patients $\geq 65$ years that attended our office from May 2013 to July 2014 with treated or untreated moderate-severe or profound sensorineural hearing loss (SNHL) were enrolled in the study and underwent further assessment. To eliminate severe cases of cognitive dysfunction, all subjects met the criteria of fewer than 3 fails on the Pfeiffer test [Pfeiffer, 1975]. Individual data sets were available for preimplantation and posttreatment intervals at a minimum of 2 years after device treatment (CI or HA) for audiometric threshold testing (pure tone before treatment and free-field using warble tones after treatment) and speech recognition tests (Spanish disyllabic words at $65 \mathrm{~dB}$ SPL in quiet). The case file reviews yielded 4 subgroups of subjects: a CI group and a corresponding control group as well as an HA group and their corresponding control group. Both control groups presented with similar age, hearing loss thresholds and hearing functionality on disyllabic word speech scores. The division of the clinical cohort into each of the 4 subgroups with summary characteristics is shown in figure 1 .

\section{Evaluation Battery}

A battery of tests was administered prospectively to evaluate the overall well-being of the patients specifically related to anxiety, depression, health status and quality of life and their perceived handicap using standardly available clinical evaluation tools. All subjects included in the study completed the tests listed in table 1. They consisted of the Geriatric Depression Scale [Yesavage et al., 1983] where a score higher than 5 indicates a suspicion of depression, the Generalized Anxiety Disorder 7 [Spitzer et al., 2006] where a score higher than 5 indicates a suspicion of an anxiety disorder, and the Hearing Handicap Inventory for the Elderly (HHIE) [Ventry and Weinstein, 1984] indicating effects of hearing loss upon daily activities. For the HHIE, a score lower than 16 points in each subscale indicates no handicap, a score from 17 to 42 means a mild to moderate handicap and a score higher than 43 means a severe handicap. The Health Utility Index mark III (HUIIII) [Francis et al., 2002] measures the multi-attribute health utility from 0 to 1.0 with 1.0 representing normal health. Contrary to the other questionnaires, the closer to 1 the score is, the better the result.

\section{Statistical Analysis}

Statistical analyses to compare score differences on each test measure between the subgroups were performed using the MannWhitney $\mathrm{U}$ test. Two-sided p values $<0.05$ were considered statistically significant. All statistical analyses were carried out with IBM SPSS Statistics version 22.

\section{Results}

A descriptive view to the performance of the treated groups is shown in figure 2. Mean percent correct disyllabic word scores, both before and after hearing device treatment (HA/CI) are shown in figure $2 \mathrm{~b}$. Significant au- 


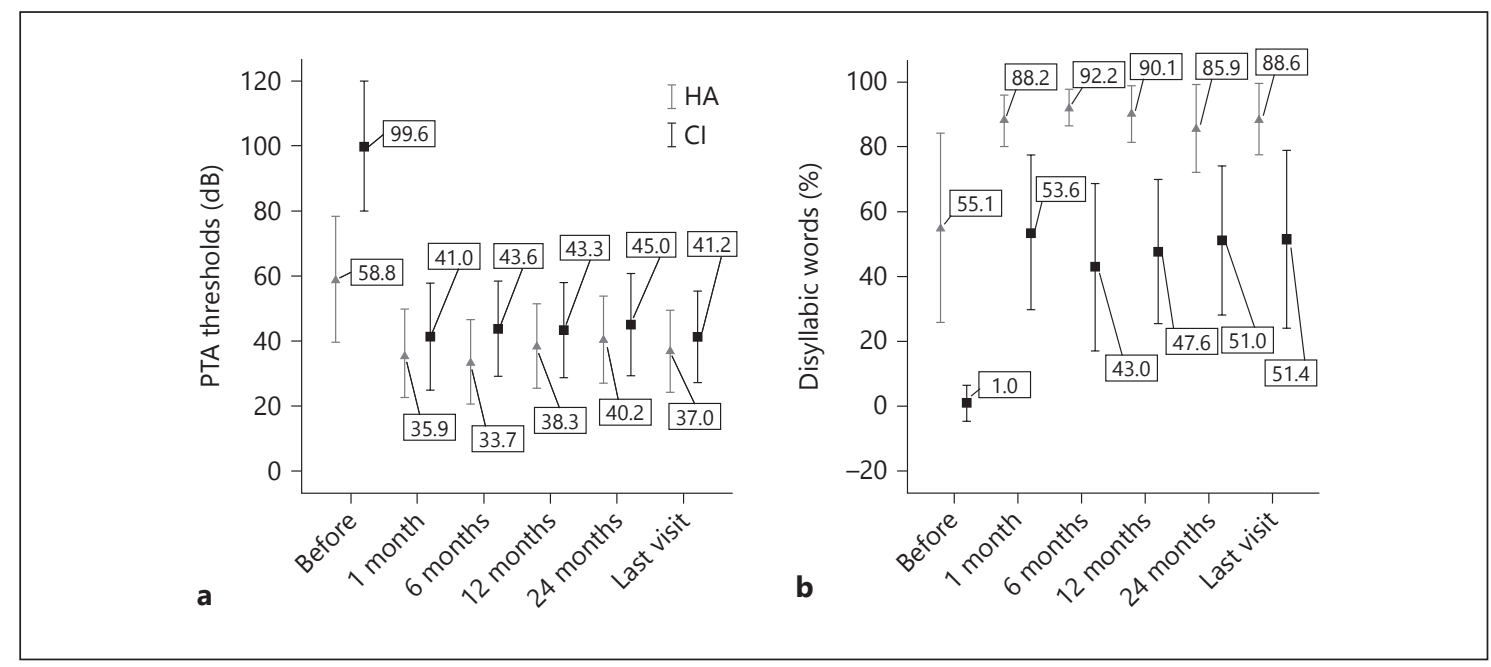

Fig. 2. Unaided and aided auditory thresholds. a Pure-tone average (PTA) thresholds $(0.5,1,2$, and $4 \mathrm{kHz})$ in both CI and HA groups, before treatment and during follow-up until the last visit. b Speech perception for disyllabic words at $65 \mathrm{~dB}$ SPL in quiet for CI and HA groups before and during follow-up until the last visit.

Table 1. The median scores and interquartile ranges are shown for each subjective questionnaire in the untreated and device-treated subgroups

\begin{tabular}{lccccc}
\hline & \multicolumn{2}{l}{ Untreated groups } & & \multicolumn{2}{l}{ Treated groups } \\
\cline { 2 - 3 } \cline { 5 - 6 } & MS-SNHL & P-SNHL & & HA & CI \\
\hline GAD-7 & $3.5(3)$ & $5(8)$ & & $3(6)$ & $2.5(4)$ \\
GDS & $1.5(3)$ & $3.5(3)$ & & $2(6)$ & $2(2)$ \\
HUI-III & $0.67(0.422)$ & $0.45(0.11)$ & & $0.61(0.39)$ & $0.57(0.33)$ \\
Total HHIE & $20(20.5)$ & $65.2(19.37)$ & & $34(34)$ & $53.32(21.41)$ \\
Emotional HHIE & $6(14)$ & $33(20)$ & & $14(13)$ & $22(20)$ \\
Situational HHIE & $13.13(7.41)$ & $32.8(10.88)$ & & $20.87(10.52)$ & $31.18(10.27)$ \\
\hline
\end{tabular}

MS-SNHL = Moderate-severe SNHL; P-SNHL = profound SNHL; GAD-7 = Generalized Anxiety Disorder 7; GDS = Geriatric Depression Scale; HUI-III = Health Utility Index mark III; HHIE = Hearing Handicap Inventory for the Elderly.

ditory function improvement is observed after treatment in both device-treated subgroups compared to their pretreatment performance level on disyllabic word tests in quiet $(\mathrm{p}<0.001)$, which remains stable over time up to 2 years and more. In figure $2 \mathrm{a}$, the significant functional gain obtained after treatment in each device subgroup is illustrated, comparing aided and unaided warble tone thresholds in the free field; the threshold also remains stable over time $(\mathrm{p}<0.001)$.

The median scores and interquartile ranges are shown for each subjective questionnaire used for treated and untreated groups in table 1 . However, a more detailed view to the distribution of scores for each of the 4 subgroups for all measures is shown in figures 3-5. Figure 3 shows the results for Generalized Anxiety Disorder 7 and Geriatric Depression Scale scores suggesting no significant differences between the device treatment subgroups and their untreated controls. Furthermore, no significant differences are noted either between the 2 device subgroups or between the 2 non-treatment control groups on these measures.

The distribution of multi-attribute health utility index scores obtained via the HUI-III (fig. 4a) indicates significantly better health utility scores for CI users compared 


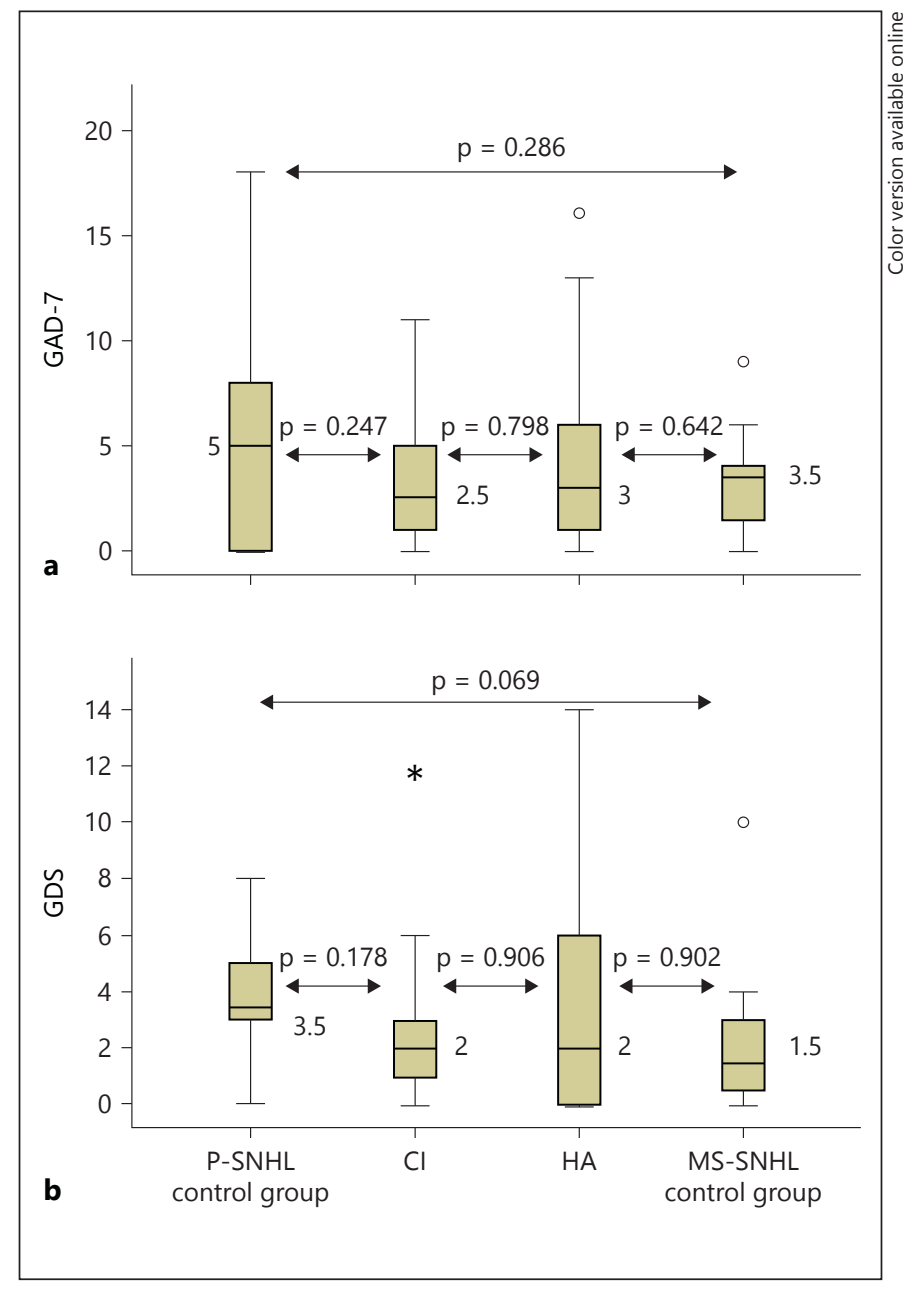

Fig. 3. a Median scores for the Generalized Anxiety Disorder 7 (GAD-7) test for every subgroup. b Median scores for the Geriatric Depression Scale (GDS) test for every subgroup. The p value corresponds to the comparison between groups. Asterisk and circles represent outliers of each group. P-SNHL = Profound SNHL; MSSNHL $=$ moderate to severe SNHL.

to their untreated control group ( $\mathrm{p}=0.014)$, while no significant differences between the HA user subgroup and their control subgroup were found. The HHIE scores shown in figure $4 \mathrm{~b}$ suggest the greatest handicap due to hearing loss is observed for the untreated profound SNHL subgroup and least for the moderate-severe SNHL group. While no significant differences were noted between treated and untreated control groups, CI users had a significantly higher handicap than the HA user group $(\mathrm{p}<$ $0.001)$.

Closer examination of the HHIE scores, dividing the responses to questions describing emotional and situational handicap, was performed for each subgroup, and

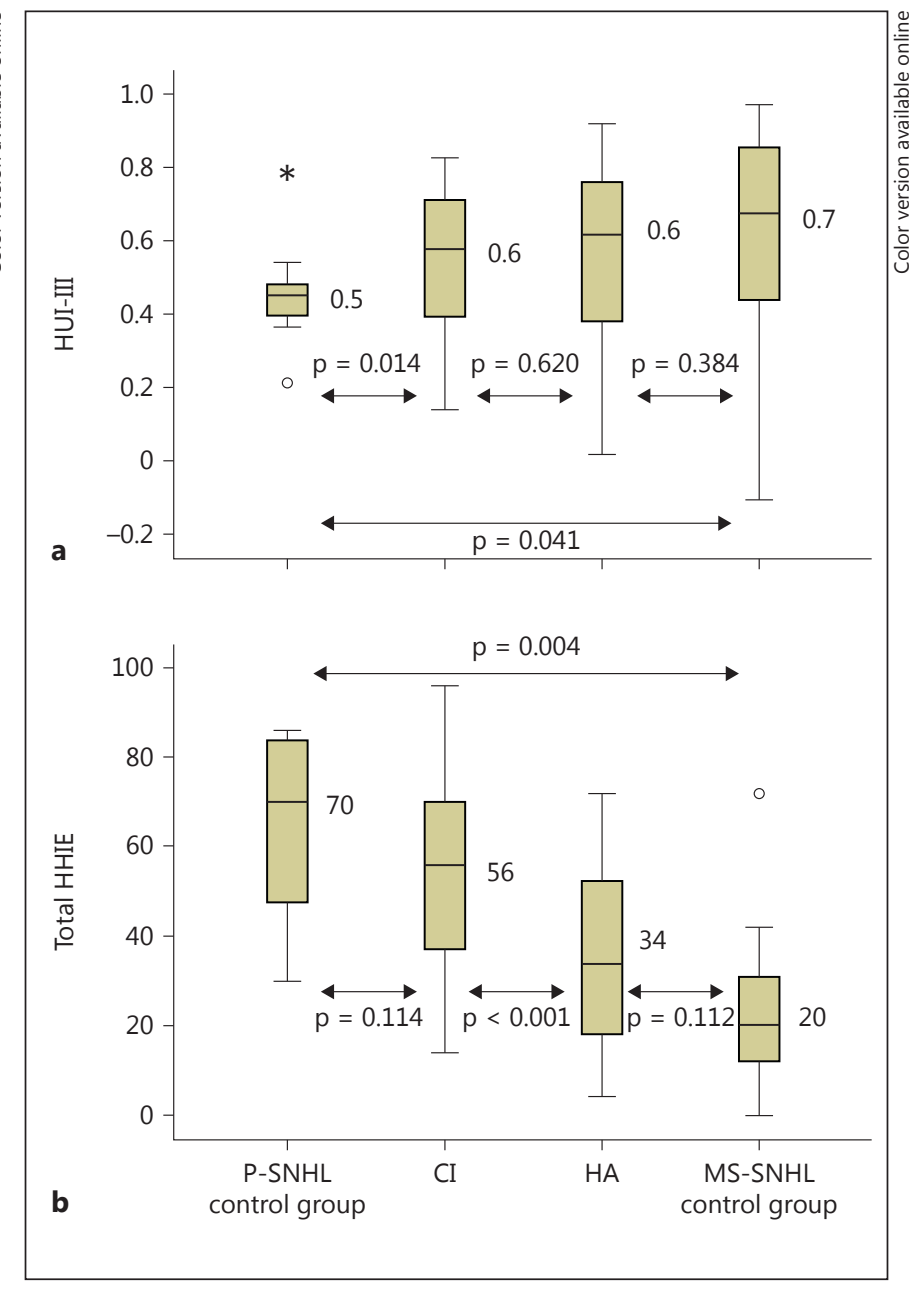

Fig. 4. a Median scores for the HUI-III test for every subgroup. b Median scores for total HHIE test scores for every subgroup. The $p$ value corresponds to the comparison between the groups. Asterisk and cirlces represent outliers of each group.

the results are illustrated in figure 5. Results suggest a trend towards decreased situational and emotional handicap for both device user groups compared to the untreated control groups; however, the difference is only statistically significant for situational handicap for the HA user group $(\mathrm{p}=0.003)$. Furthermore, the differences for both situational and emotional handicaps are statistically significant between untreated groups and between device user groups. Not surprisingly, untreated profound SNHL and CI users experience a significantly greater handicap than the untreated moderate-severe SNHL group and the HA users, respectively $(\mathrm{p}<0.001)$. 


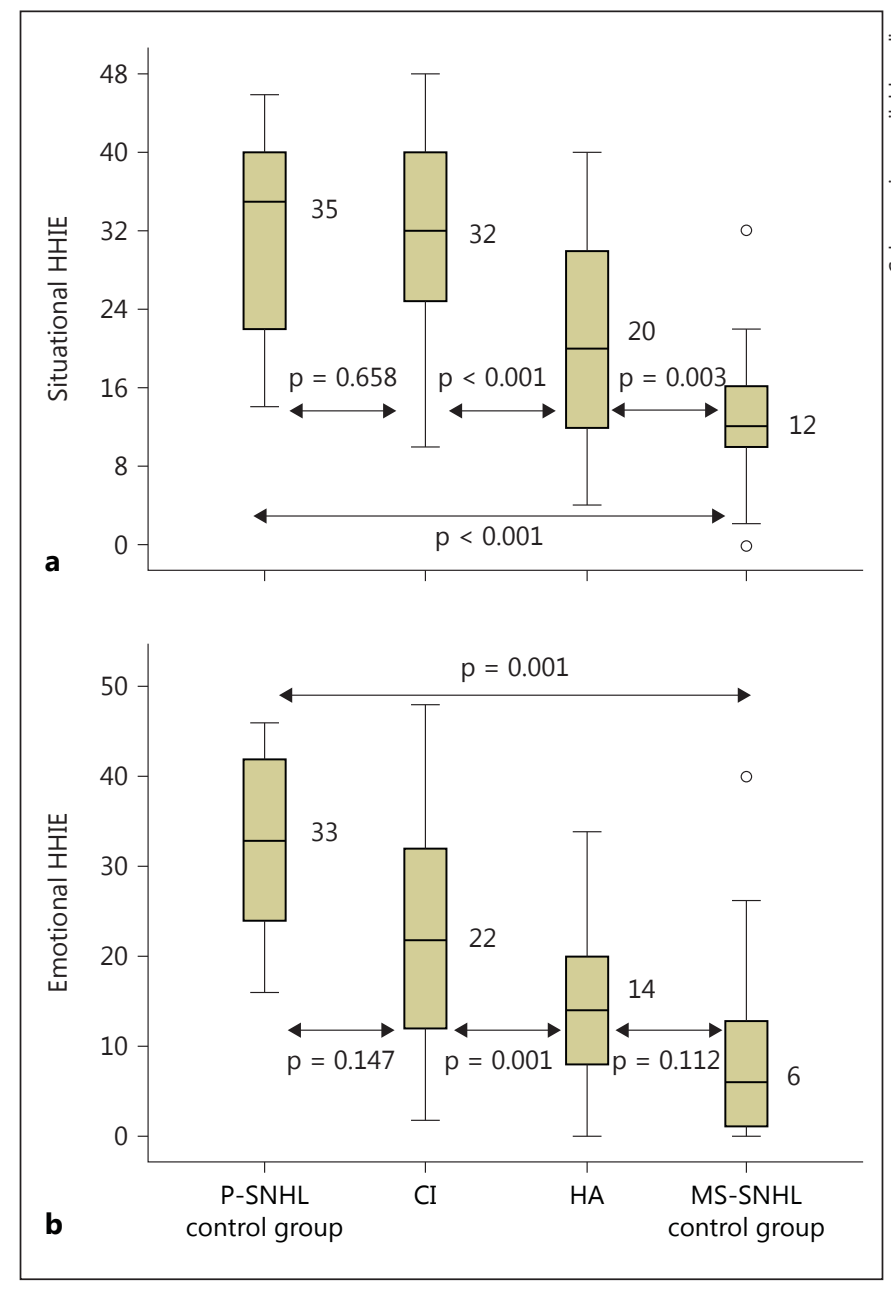

Fig. 5. a Median scores for situational HHIE for every subgroup. b Median scores for emotional HHIE for every subgroup. The $\mathrm{p}$ value corresponds to the comparison between groups. Circles represent outliers of each group.

\section{Discussion}

This study confirms that the application of HA or CI is useful in reducing hearing disability in patients older than 65 years experiencing hearing loss, depending on the degree of hearing loss. This benefit is demonstrated by improvements in hearing thresholds, disyllabic word recognition scores, multi-attribute HUI values and hearing handicap emotional and situational scores.

The use of conventional amplification for moderate to severe SNHL can improve speech recognition significantly as illustrated for our study cohort of HA users with scores increasing from $55 \%$ unaided to $88 \%$ aided after 6 months of follow-up. However, the proportion of hearing-impaired people who actually wear an HA, even in developed countries, is still relatively low where more than half do not use any amplification [Popelka et al., 1998]. Some reasons that may explain the poor usage of HAs have been described by Gopinath et al. [2011] and include expensive costs of HA, social stigma with patients embarrassed to wear them, or the absence or lack of proper information or guidance on HA use. The end result is that the majority of patients older than 65 years with moderate to severe SNHL are not being properly treated.

As shown by our study results, the degree of hearing loss alone (despite the age and potential for other comorbidities) carries an impact on daily life and communication. The more profound the degree of hearing loss, the more limited the patient becomes. Surprisingly, after fitting an HA, patients may have a poorer perception of their hearing ability and its subsequent limitations than prior to the HA fitting. Possibly after the fitting of an HA, the individuals may become more aware of their hearing impairment and the social and communication limitations and consequences. In contrast, Vuorialho et al. [2006] described that using HAs correctly has been reported to lead to decreased hearing handicap scores when questioned with the HHIE. Such changes in their study are shown even when non-users (10\%) are included. They conclude that HA fitting, as a process, has a strong positive impact on emotional states.

The moderate-severe SNHL groups (treated and nontreated) show no differences in anxiety and depression scores. Such findings contrast with previous reports that stated that high HHIE scores are a risk factor in the development of depression amongst elderly individuals [Saito et al., 2010]. Our analysis of HHIE scores was derived from cohorts of treated and untreated individuals within a hearing-impaired population, whereas Saito et al. [2010] compared normal-hearing subjects to hearingimpaired ones. Thus, such differences in the incidence of depression between both groups may be related to the population studied. Our recommendation is to include an assessment of the perceived handicap via the HHIE as a useful screening tool for community-dwelling elderly people to identify those at risk.

Our study clearly demonstrates greater benefit from cochlear implantation for older adults with more significant hearing loss than from conventional amplification for the HA user group. Postimplantation, audiometric tests suggest significant improvements in auditory thresholds over the pre-operative unaided condition and for mean word recognition scores on disyllabic words, which 
reached $52 \%$ compared to $0 \%$ before implantation. As shown in figure 2, the largest and most rapid improvement in hearing performance was observed within the first 6 months of treatment, remaining stable thereafter. Previous studies have shown that the learning curve for elderly implantees is similar to that observed for the younger implanted population, while absolute scores for the elderly measured in noisy environments tend to be lower [Lenarz et al., 2012]. Our cohort demonstrated that once the rapid auditory improvement is seen, auditory outcomes increase slowly, reaching a plateau independent of age and is maintained during the follow-up. This may be due to the fact that neural degeneration might be prevented with auditory stimulation [Leake et al., 2008]. Several factors may influence benefits from CI treatment in older adults that include pre-operative speech discrimination [Lin et al., 2012], overall status of health [Clark et al., 2012], consistency of HA use within a lifetime, educational level, residential status and level of depression [Francis et al., 2015].

Given it is common audiological practice to demonstrate that $\mathrm{CI}$ and HA treatment can provide adequate functional gain for the respective groups of hearing-impaired elderly patients, through improved speech recognition, we can assume a logical consequence is that communication is also improved; however, the question arises: what is the ultimate impact on their daily life?

According to data for our study cohort, anxiety and depression levels show no correlation with treated or untreated conditions or the degree of hearing loss, whereas a trend for lower levels of anxiety and depression are noted in the CI group compared to their control group. In a study by Olze et al. [2012], it was demonstrated that hearing loss significantly influences isolation, which explains the influence in the social domain.

Based on our findings from the HUI-III test, the degree of hearing loss can positively influence the perception of one's overall health status. This is supported by our observation that the CI group reported a significantly greater improvement in the perception of their health status (multi-attribute HUI) and, thus, quality of life than the control group for quality of life. However, in contrast, there was no significant difference between the reported health utility for HA users compared to the untreated control group.

This study demonstrates that the influence of hearing disability upon daily life increases with increased hearing loss. As expected, patients with profound hearing loss endure more limitations in their daily lives due to the hearing loss than patients with a moderate-severe hearing loss.
The negative consequences of hearing loss on quality of life and perceived handicap demonstrated in our cohort suggest that timely and appropriately administered sound amplification can potentially mitigate the resounding effects of hearing loss for the individual. These findings agree with data published by Lin et al. [2012] stating that hearing loss may potentially increase the risk of depression and social isolation due to communication impairment. They propose that significant hearing loss may provoke further cognitive decline through the inherent cognitive load that, in turn, can negatively influence physical and daily functioning. As social isolation and cognitive load increase and physical conditions decrease, the risk of dementia and incidence of morbiditymortality can increase. These findings are confirmed by research demonstrating that significantly reduced auditory input is associated with cognitive dysfunction and decline and, in extreme cases, with age-related dementia [Kricos, 2006]. Furthermore, it has been demonstrated that good cognitive function is extremely important in processing auditory information when made available via hearing treatment [Mosnier et al., 2015]. Therefore, not only does hearing loss have a negative impact on cognitive function, cognitive impairment has a negative impact on hearing function. While not causative, hearing loss is also an unrelated risk factor for dementia linked to a characteristic auditory pattern [Gimeno-Vilar and Cervera-Paz, 2012].

\section{Conclusion}

Our study has shown that hearing treatment with a CI or HA can provide significant advantages to hearing function that remains stable over time in elderly patients. Significant improvements for CI patients with profound SNHL are observed for quality of life and show a trend towards reduced perceived hearing handicap. Patients with an untreated profound hearing loss demonstrate a significantly poorer quality of life and show a trend for higher levels of anxiety, depression and the perceived handicap than comparable patients treated with a CI. In contrast, the significant effects beyond hearing function were not demonstrated between HA users and their respective control group with untreated moderate-severe hearing loss. In view of the inherent progressive nature of presbycusis and the observed relation between significantly reduced auditory input upon socio-emotional and overall health domains, hearing loss should always be treated in adults older than 65 years.
Manrique-Huarte/Calavia/Huarte Irujo/ Girón/Manrique-Rodríguez 


\section{Acknowledgements}

Special thanks go to Salome Sanbruno and Charo Lezaun, the nurses who helped in recruiting patients and assisted with completing the tests.

The study was funded by the grant Juan Gassó Bosch.

\section{Disclosure Statement}

The authors declare no conflict of interest.

\section{References}

Clark J, Yeagle J, Arbaje A, Lin F, Niparko J, Francis $\mathrm{H}$ : Cochlear implant rehabilitation in older adults: literature review and proposal of a conceptual framework. JAGS 2012;60:19361945.

Francis HW, Chee N, Yeagle J, et al: Impact of cochlear implant on the functional health status of older adults. Laryngoscope 2002;112: $1482-1488$.

Francis HW, Yeagle J, Thompson C: Clinical and psychosocial risk factors of hearing outcome in older adults with cochlear implant. Laryngoscope 2015;125:695-702.

Gimeno-Vilar C, Cervera-Paz FJ: Enfermedad de Alzheimer y pérdida auditiva. Rev Neurol 2010;50:65-71.

Gopinath B, Schneider J, Hartley D, Teber E, McMahon CM, et al: Incidence and predictors of hearing aid use and ownership among older adults with hearing loss. Ann Epidemiol 2011; 21:497-506.

Kricos PB: Audiologic management of older adults with hearing loss and compromised cognitive/psychoacoustic auditory processing capabilities. Trends Amplif 2006;10:1-28.

Leake PA, Stakhovskaya O, Hradek GT, Hetherington AM: Factors influencing neurotrophic effects of electrical stimulation in the deafened developing auditory system. Hear Res 2008;242:86-99.
Lenarz M, Sönmez H, Joseph G, Büchner A, Lenarz T: Cochlear implant performance in geriatric patients. Laryngoscope 2012;122: 1361-1365.

Lin F, Chien W, Li L, Clarret D, Niparko J, Fancis $\mathrm{H}$ : Cochlear implant in older adults. Medicine 2012;91:229-241.

Mosnier I, Bebear JP, Marx M, Fraysse B, Truy E, Lina-Granade G, Mondain M, Sterkers-Artières F, Bordure P, Robier A, Godey B, Meyer B, Frachet B, Poncet-Wallet C, Bouccara D, Sterkers O: Improvement of cognitive function after cochlear implantation in elderly patients. JAMA Otolaryngol Head Neck Surg 2015;141:442-450.

Olze H, Gräbel S, Förster U, Zirke N, Huhnd LE, Haupt H, Mazurek B: Elderly patients benefit from cochlear implant regarding auditory rehabilitation, quality of life, tinnitus and stress. Laryngoscope 2012;122:196-203.

Pfeiffer E: A short portable mental status for the assessment of organic brain in elderly patients. J Am Geriatr Soc 1975;23:433-441.
Popelka MM, Cruickshanks KJ, Wiley TJ, Tweed TS, Klein BE, et al: Low prevalence of hearing aid use among older adults with hearing loss: the Epidemiology of Hearing Loss Study. J Am Geriatr Soc 1998;46:1075-1078.

Saito H, Nishiwaki Y, Michikawa T, Kikuchi Y, Mizutari K, et al: Hearing handicap predicts the development of depressive symptoms af ter 3 years in older community-dwelling Japanese. J Am Geriatr Soc 2010;58:93-97.

Spitzer RL, Kroenke K, Williams JB, Löwe B: A brief measure for assessing generalized anxiety disorder: the GAD-7. Arch Intern Med 2006;166:1092-1097.

Sprinzl GM, Riechelmann H: Current trends in treating hearing loss in elderly people: a review of the technology and treatment options - a mini review. Gerontology 2010;56: 351-358.

Ventry IM, Weinstein BE: The hearing handicap inventory for the elderly: a new tool. Ear Hear 1984;3:128-134.

Vuorialho A, Karinen P, Sorri M: Effect of hearing aids on hearing disability and quality of life in the elderly. Int J Audiol 2006;45:400-405.

Yesavage JA, Brink TL, Rose TL, Lum O, Huang V, Adey MB, Leirer VO: Development and validation of a geriatric depression screening scale: a preliminary report. J Psychiatr Res 1983;17:37-49. 